\title{
Monitoring Machines by Using a Hybrid Method Combining MED, EMD, and TKEO
}

\author{
Mourad Kedadouche, Marc Thomas, and Antoine Tahan \\ Department of Mechanical Engineering, École de Technologie Supérieure, 1100 Notre-Dame Street West, \\ Montreal, QC, Canada H3C $1 \mathrm{~K} 3$ \\ Correspondence should be addressed to Marc Thomas; marc.thomas@etsmtl.ca
}

Received 17 September 2013; Revised 11 December 2013; Accepted 7 January 2014; Published 20 March 2014

Academic Editor: Joseph C. S. Lai

Copyright (C) 2014 Mourad Kedadouche et al. This is an open access article distributed under the Creative Commons Attribution License, which permits unrestricted use, distribution, and reproduction in any medium, provided the original work is properly cited.

\begin{abstract}
Amplitude demodulation is a key for diagnosing bearing faults. The quality of the demodulation determines the efficiency of the spectrum analysis in detecting the defect. A signal analysis technique based on minimum entropy deconvolution (MED), empirical mode decomposition (EMD), and Teager Kaiser energy operator (TKEO) is presented. The proposed method consists in enhancing the signal by using MED, decomposing the signal in intrinsic mode functions (IMFs) and selects only the IMF which presents the highest correlation coefficient with the original signal. In this study the first $\mathrm{IMF}_{1}$ was automatically selected, since it represents the contribution of high frequencies which are first excited at the early stages of degradation. After that, TKEO is used to track the modulation energy. The spectrum is applied to the instantaneous amplitude. Therefore, the character of the bearing faults can be recognized according to the envelope spectrum. The simulation and experimental results show that an envelope spectrum analysis based on MED-EMD and TKEO provides a reliable signal analysis tool. The experimental application has been developed on acoustic emission and vibration signals recorded for bearing fault detection.
\end{abstract}

\section{Introduction}

The bearing may be considered as one of the most stressed parts in rotating machines. Early stage bearing defects excite first the resonance frequencies which manifest in the high frequency domain. High frequency resonance technique (HFRT) is thus mostly used in industry since it allows the extraction of components information representing defects on rotating machinery [1]. A band-pass filtering around the excited resonance frequency followed by an amplitude demodulation step exhibits the modulation frequencies representative of the fault characteristic frequency of the bearing and its associated harmonics [2,3]. Spectral analysis of this signal (amplitude and number of harmonics) can reveal the severity of defects [4]. However, the major challenge in the application of the HFRT technique is the proper selection of the center frequency and bandwidth of the band-pass filter. Many researches have focused on the development of efficient and robust methods for estimating the proper center frequency and optimum bandwidth of the band-pass filter.
Spectral Kurtosis has been proposed by Antoni and Randall [5]. However Kurtosis has its own limitation, especially when the signal is submerged by a strong and non-Gaussian noise with sudden high peaks where kurtosis shows extremely high values [6]. Other methods were developed. Barszcz and Jabłoński [7] proposed a Portrugram while Qiu et al. $[8,9]$ used the minimal Shannon entropy to select the proper bandwidth. Nikolaou and Antoniadis [10] and Lin and Zuo [11] selected the parameters of Morlet wavelet based on the kurtosis maximization criterion. The most commonly used method to estimate the amplitude envelope of a signal is based on the analytic signal which is derived using the Hilbert transform.

On the other hand, teager energy operator (TEO) [12], originally proposed for nonlinear signal processing, provides an effective approach to extract the instantaneous amplitudes and frequencies of a modulated signal by energy operator demodulation approach and the demodulation effect is good compared with Hilbert demodulation method; meanwhile the computation time greatly decreases [13-17]. This method 
was first used for the frequency and amplitude demodulation of the speech signals [12-15] and was recently adopted for vibration signals of rotating machines [16]. It incorporates both amplitude and frequency demodulation and improves the fault signal to interference ratio and thus could enhance detection performance. The energy separation algorithm is completely data driven without the need to construct any basis functions. It is therefore adaptive to the local structure of a signal. It can track the energy and identify the instantaneous frequency and instantaneous amplitude of monocomponent signals. However, it is well known that the vibration or acoustic emission signal from bearings is a multicomponent signal. For this reason, before computing TKEO, we propose to decompose the signal in multicomponents by using the empirical mode decomposition (EMD) method, since it can adaptively decompose the signal into intrinsic mode functions (IMFs) [18-22]. The TKEO can be computed for the IMF which is the most correlated with the original signal [23-25]. We thus propose to use the coefficient of correlation as an indicator to select the IMF. So, the selected IMF is the IMF which presents the higher correlation coefficient with the original signal.

However, in the early stages of bearing failure, the signal amplitude is somewhat weak and is often hidden by large noises and other signals. The selected IMF may thus be submerged by noise and cannot effectively discriminate the real bearing signal from noise. Furthermore, the TKEO is highly sensitive to the noise level. A preprocessing method is thus needed for signal decomposition using the EMD method. We propose to use the minimum entropy deconvolution (MED) which has been proven to be efficient in detecting incipient faults buried in large noise and allows for an optimal filter to extract faulty bearing signals $[26,27]$. Applying the EMD method to the filtered signal allows for the signal feature of the faulty bearing to be adaptively separated from the remaining noise.

We thus proposed to combine all these three methods for the diagnosis of bearing faults. The simulation and experimental results show that this hybrid method can effectively diagnose the faults of the bearing faults. The paper is organized as follows. Section 2 introduces the principles of EMD and its algorithm. Section 3 presents the TKEO theory. Section 4 presents the basic principles of MED. Section 5 gives the results from simulation and experimental data of bearing faults. Finally, the conclusions are provided in Section 6 .

\section{Empirical Mode Decomposition (EMD)}

The EMD method decomposes the time signal into a finite set of oscillatory functions called intrinsic mode functions (IMF). An IMF is a function that meets the following conditions.

(i) The number of extrema and the number of zero crossings must either be equal or differ at most by one.

(ii) The value of the moving average envelope defined by local maxima and the envelope defined by local minima is zero.
The decomposition method in IMF may be summarized as follows [19-22].

(1) Initialize: $r_{0}=x(t)$, and $i=1$.

(2) Extract the $i$ th IMF $c_{i}$

(a) initialize: $h_{i(k-1)}=r_{(i-1)}, k=1$;

(b) extract the local maxima and minima of $h_{i(k-1)}$;

(c) interpolate the local maxima and the minima by cubic spline lines to form upper and lower envelops of $h_{i(k-1)}$;

(d) calculate the mean $m_{i(k-1)}$ of the upper and lower envelops of $h_{i(k-1)}$;

(e) let $h_{i k}=h_{i(k-1)}-m_{i(k-1)}$;

(f) if $h_{i k}$ is an IMF, then set $c_{i k}=h_{i k}$; else go to step (b) with $k=k+1$

(3) Define the remainder $r_{i+1}=r_{i}-c_{i}$.

(4) If $r_{i+1}$ still has least 2 extrema, then go to step (2) with $i=i+1$; else, the decomposition process is finished and $r_{i+1}$ is the residue of the signal.

\section{Teager Operator Energy}

The Teager-Kaiser energy tracking operator (TKEO) is a nonlinear operator considered as a high-resolution energy estimator quantifying a product of both frequency and amplitude. TKEO can track the modulation energy and identify the instantaneous amplitude and frequency. This operator was developed by Kaiser [12]. TKEO, applied on a continuous signal $x(t)$ is defined as

$$
\Psi(x(t))=[\dot{x}(t)]^{2}-x(t) \ddot{x}(t) .
$$

The discrete function equivalent to the energy operator is given by

$$
\Psi(x(n))=[x(n)]^{2}-x(n+1) x(n-1),
$$

where $x(n)$ is a discrete-time signal.

The energy operator can extract the amplitude modulation and the frequency modulation from the signal. The TKEO has a great time resolution since the operator only needs three samples into the signal to be computed. This excellent time resolution provides the ability to capture the energy fluctuations. Furthermore, this operator is very easy to implement efficiently. The energy separation algorithm (ESA) developed by Maragos et al. $[14,15]$ uses the TKEO to separate $x(t)$ into its amplitude envelope $|a(t)|$ and signal $f(t)$ to accomplish monocomponent AM-FM signal demodulation:

$$
\begin{gathered}
f(t) \approx \frac{1}{2 \pi} \sqrt{\frac{\Psi(\dot{x}(n))}{\Psi(x(n))}}, \\
|a(t)| \approx \frac{\Psi(x(n))}{\sqrt{\Psi(\dot{x}(n))}} .
\end{gathered}
$$

The ESA is a very simple demodulating technique for AM-FM demodulation. It presents a good time resolution 
but is very sensitive to noise. For this reason, minimum entropy deconvolution (MED) is proposed to extract the fault impulses while minimizing the noise before computing EMD and the TKEO.

\section{Minimum Entropy Deconvolution (MED)}

MED was originally proposed for applications on seismic recordings by Wiggins since 1978 [28]. The MED is a deconvolution aimed for extracting the fault impulses while minimizing the noise [29]. Higher entropy corresponds to a tendency to become random and the aim of MED is to enhance the structured information into the signal by searching for an optimum set of filter coefficients that recover the output signal with the maximum value of kurtosis. Kurtosis is an indicator that reflects the "peakiness" of a signal and therefore the property of impulses

$$
\text { Kurtosis }=\frac{(1 / N) \sum_{i=1}^{N}\left(x_{i}-\bar{x}\right)^{4}}{\mathrm{RMS}^{4}} .
$$

The detailed steps of this implementation can be recalled from [27].

\section{Bearing Faults Diagnosis Based on MED-EMD and TKEO}

5.1. Proposed Method for Defect Detection of Ball Bearings. The choice of the IMFs to be analyzed is usually realized by visual or experience criteria by the user. However, the process is not automatic in this way and an interaction with the user is required. We propose to use the coefficient of correlation as an indicator to select the IMF. So, the selected IMF is the IMF which presents the higher correlation coefficient with the original signal.

The procedure of the proposed method based on MEDEMD and TKEO is given as follows.

(1) Enhance the peakiness of the signal by using the MED method.

(2) Decompose the time domain signal $x(t)$ into IMFs using EMD.

(3) Select the IMF which has the higher correlation coefficient.

(4) Compute the instantaneous amplitude using TKEO.

(5) Analyze the spectrum of the envelope computed.

In order to demonstrate the effectiveness of the proposed method for fault detection of ball bearings, we proposed to compare between three methods.

(i) The first method consists in applying the TKEO to the original signal $x(t)$.

(ii) The second method consists in decomposing the signal $x(t)$ into IMFs, selecting the IMF with higher correlation coefficient, and computing the TKEO (see Figure 1).

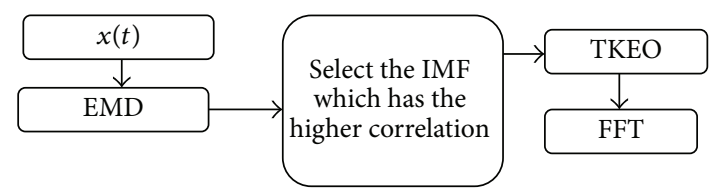

FIGURE 1: Method 2 (without using MED).

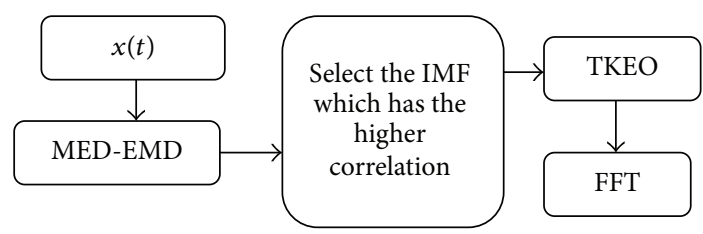

FIGURE 2: Method 3 (using MED before applying EMD).

(iii) The last one is the proposed method: step 1 consists in enhancing the peakiness of the signal using the MED technique; after that, decomposing the signal into IMFs, selecting the IMF with higher correlation coefficient, and, finally, computing the TKEO (see Figure 2).

5.2. Numerically Simulated Signal. The numerically simulated signal that has been chosen is similar to the signal used in [30] with added noise. Its mathematic formulation is given as

$$
\begin{gathered}
x(t)=e^{-\alpha \grave{t}}\left(A \sin \left(2 \pi f_{1} t\right)+B \sin \left(2 \pi f_{2} t\right)\right)+n(t), \\
\grave{t}=\operatorname{mode}\left(t, \frac{1}{F_{m}}\right), \\
n(t) \sim N(0,0.3),
\end{gathered}
$$

where $\alpha$ is equal to $900, F_{m}$ is the bearing fault frequency (BPFO equal to $100 \mathrm{~Hz}$ ), $A=0.1$, and $B=0.8 . F_{s}$ is the sampling frequency set to $48000 \mathrm{~Hz}$. $f_{1}$ and $f_{2}$ are the two resonant frequencies (equal to $1800 \mathrm{~Hz}$ and $4000 \mathrm{~Hz}$, resp.).

A normally distributed random signal with 0 mean and standard deviation of 0.3 is added into the simulated signal in (6). The signal $x(t)$ is shown in Figure 3.

5.2.1. Method 1: TKEO Applied to $x(t)$. Computing the TKEO for the raw signal exhibits good results. Figure 4 exhibits clearly the peaks of BPFO and its harmonics which are representative of the defect.

5.2.2. Method 2: Applying EMD and TKEO. For faulty bearing, the raw signal $x(t)$ was processed using the EMD technique. Figure 5 shows the signal $x(t)$ and its IMFs and Table 1 list the Pearson correlation coefficients between the signal and their corresponding IMFs. Table 1 shows that the first three IMFs have much higher correlation coefficients than the others. Thus, Figure 5 only shows these first three IMFs. It may be noticed that most of the noise imbedded in 
TABLE 1: Correlation value between each IMF and $x(t)$.

\begin{tabular}{lccccccccccc}
\hline IMF1 & IMF2 & IMF3 & IMF4 & IMF5 & IMF6 & IMF7 & IMF8 & IMF9 & IMF10 & IMF 11 \\
\hline $\mathbf{0 . 6 5}$ & 0.45 & 0.47 & 0.22 & 0.17 & 0.12 & 0.07 & 0.06 & 0.04 & 0.03 & 0.01 \\
\hline
\end{tabular}

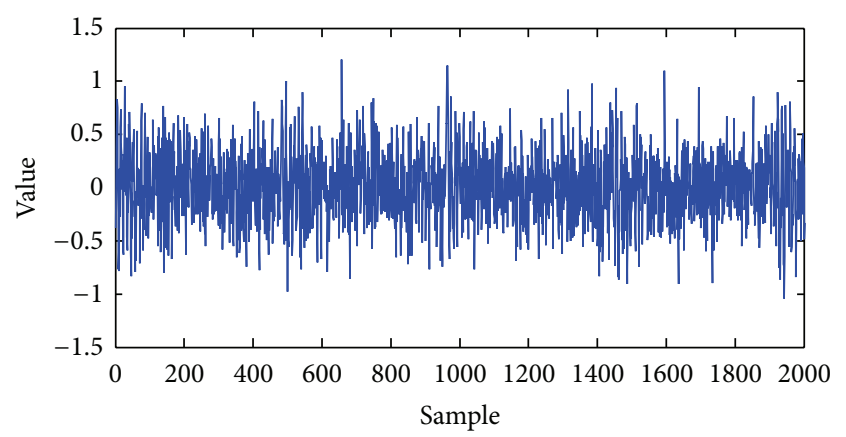

FIGURE 3: Simulated noisy signal $x(t)$.

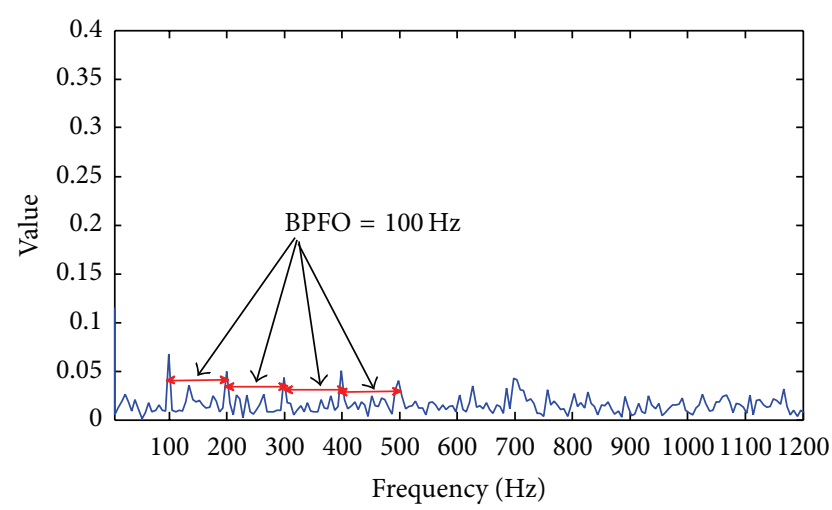

FIgURE 4: Spectrum of TKEO of $x(t)$.
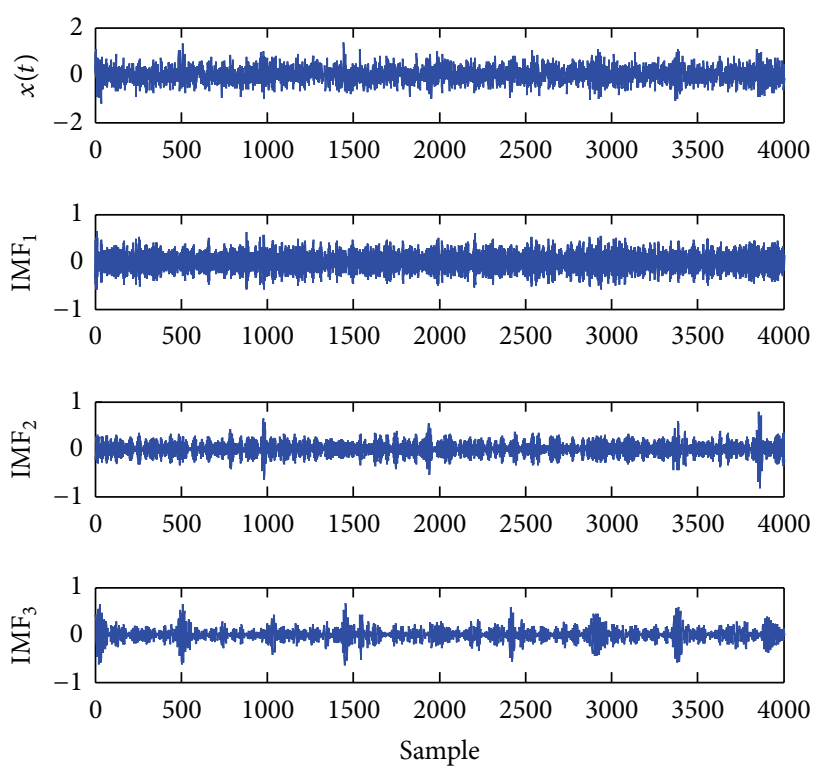

Figure 5: IMF of signal $x(t)$.

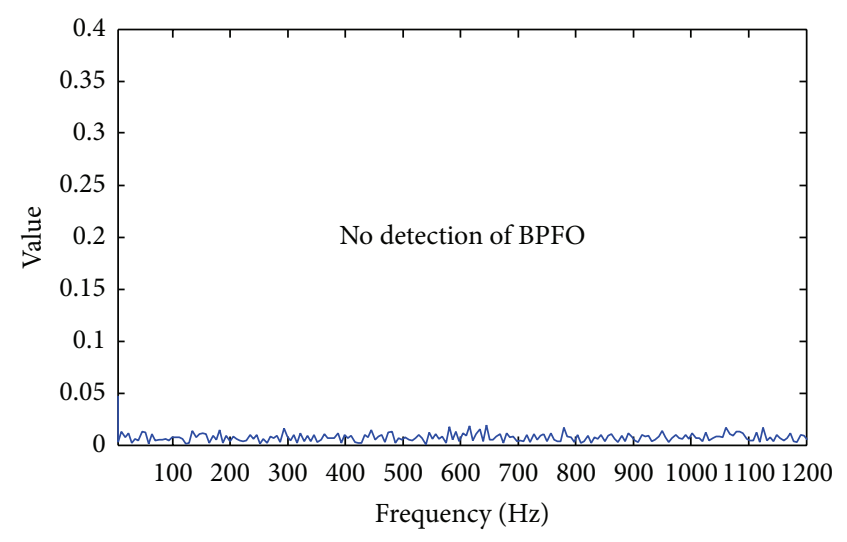

FIgURE 6: Spectrum of TKEO of IMF $_{1}$ obtained by applying EMD to $x(t)$.

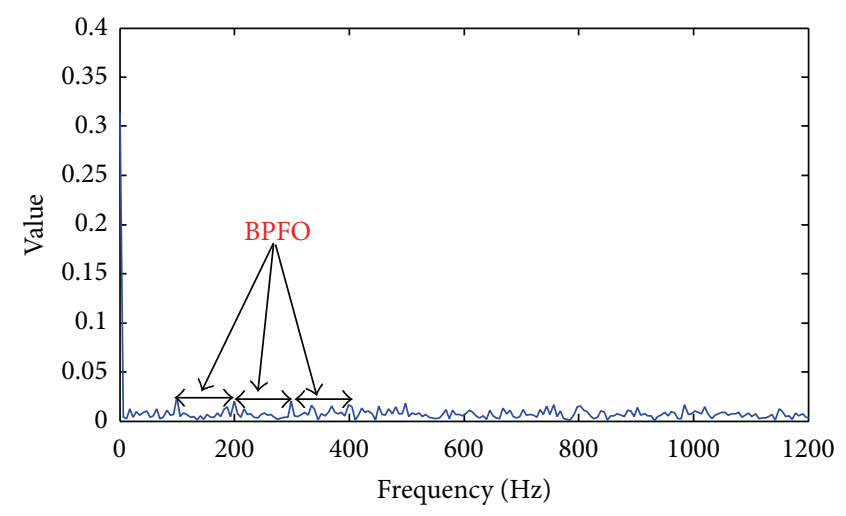

FIgURE 7: Spectrum of TKEO of $\mathrm{IMF}_{2}$ obtained by applying EMD to $x(t)$.

the raw signal resides in $\mathrm{IMF}_{1}$. The periodic impulses are still masked by noise and distributed in $\mathrm{IMF}_{2}$ and $\mathrm{IMF}_{3}$.

However, the aim of the method is only to use the IMF which has the higher coefficient value with the raw signal, in order to automatically select it.

The first $\mathrm{IMF}_{1}$ is in this case the component with the higher correlation value. The spectrum of TKEO for $\mathrm{IMF}_{1}$ is presented in Figure 6. We note a flat spectrum, which is due to the fact that the first $\mathrm{IMF}_{1}$ is very noisy, and the information related to the defect is not revealed.

The spectrum of TKEO of $\mathrm{IMF}_{2}$ and $\mathrm{IMF}_{3}$ is presented in Figures 7 and 8, respectively. It can be noticed that $\mathrm{IMF}_{3}$ exhibits the peaks of BPFO and its harmonics better than $\mathrm{IMF}_{2}$, but not very clearly. We must conclude that the method 2 is not enough efficient. 


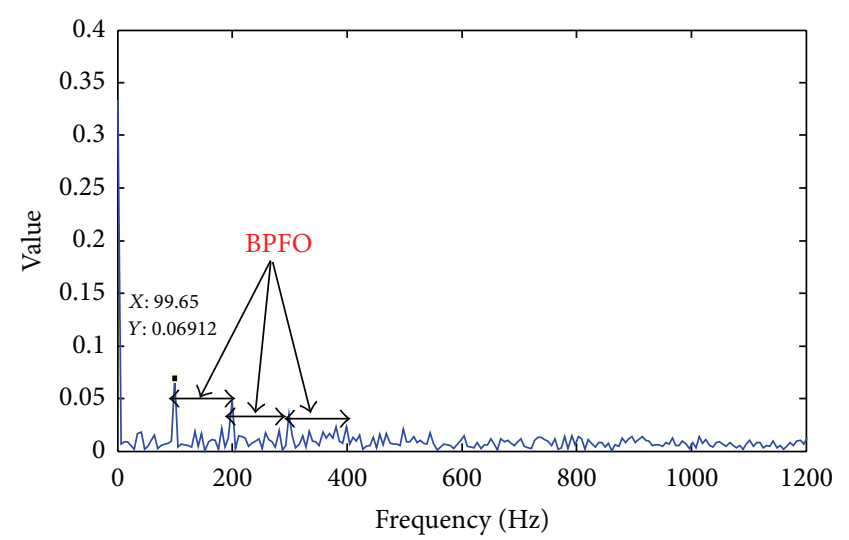

FIGURE 8: Spectrum of TKEO of $\mathrm{IMF}_{3}$ obtained by applying EMD to $x(t)$.

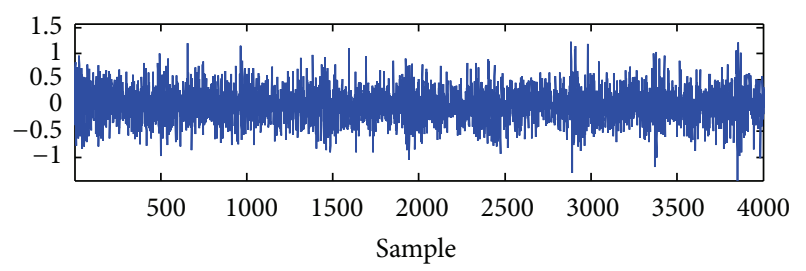

(a)

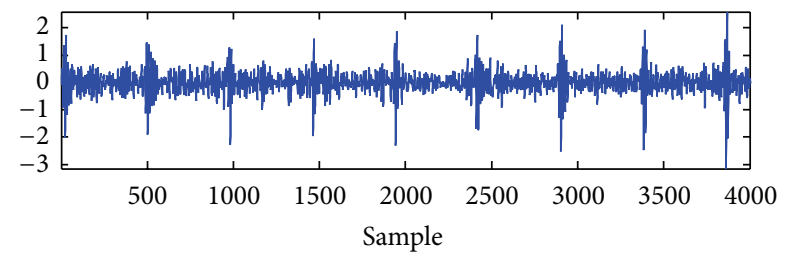

(b)

Figure 9: (a) Signal $x(t)$, (b) signal $y(t)$ obtained after applying MED.

5.2.3. Method 3: $M E D+E M D+T K E O$. Before computing EMD, the MED which maximizes the kurtosis is used in order to enhance the signal. Figure 9(b) shows the result $y(t)$ obtained after applying MED to $x(t)$. A decrease of noise may be noticed and the periodic shocks appear clearly. This result is obtained after 30 iterations when using MED. Figure 10 shows the evolution of the kurtosis value at each iteration. At the end of execution of MED, it can be noticed that the kurtosis of $y(t)$ is higher than the original signal $x(t)$.

When applying the EMD to the results obtained by MED $(y(t))$, the correlation is improved. Table 2 lists the Pearson correlation coefficients between the signal and their corresponding IMFs.

The first three IMFs exhibit much higher correlation coefficients than the others, and thus Figure 11 only shows the first three IMFs for the faulty bearings. Most of the noise is distributed into $\mathrm{IMF}_{2}$, and only the periodic impulses reside into $\mathrm{IMF}_{1}$. As indicated in Table 2, IMF $\mathrm{IM}_{1}$ has a stronger correlation coefficient (0.86) than all the other signal components and contains the main component in the filtered

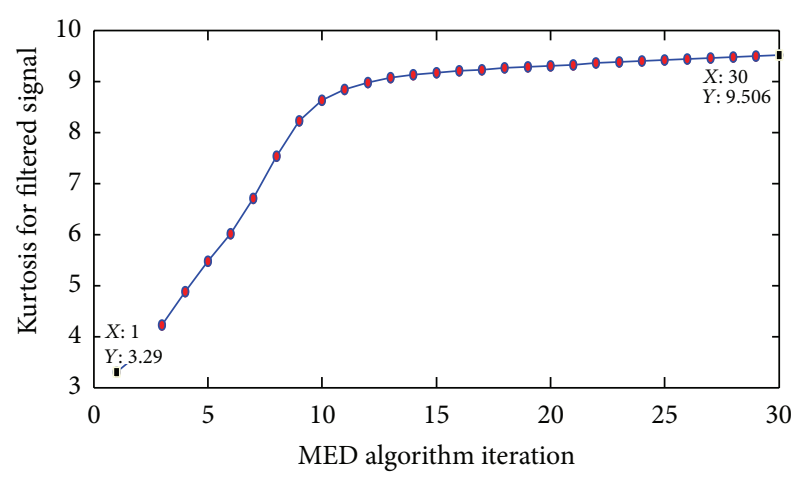

FIGURE 10: Values of kurtosis on each iteration of MED Algorithm.
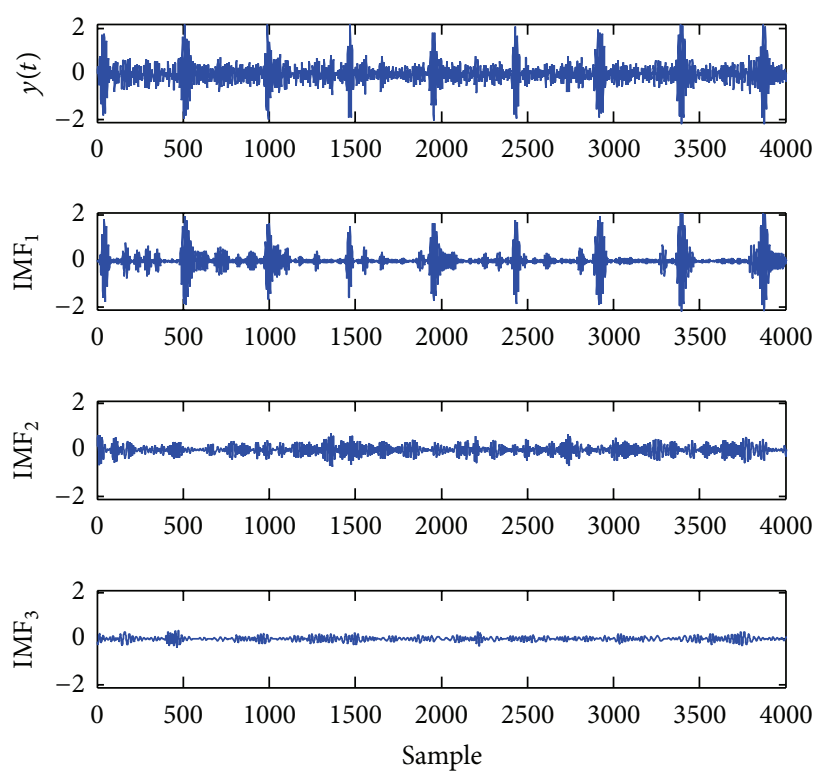

Figure 11: MED-EMD of signal.

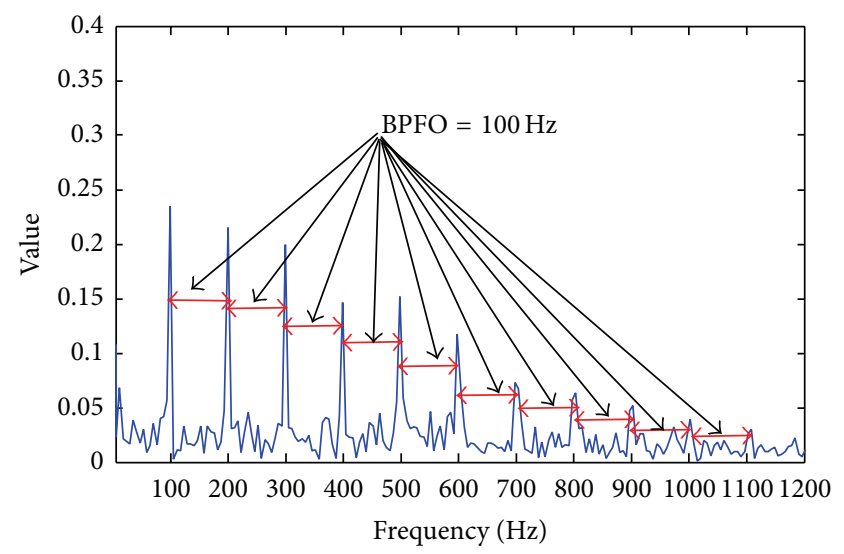

FIGURE 12: Spectrum of TKEO of IMF $_{1}$ obtained by applying MEDEMD to $x(t)$. 
TABLE 2: Correlation value between each IMF and $y(t)$.

\begin{tabular}{lccccccccccc}
\hline & IMF1 & IMF2 & IMF3 & IMF4 & IMF5 & IMF6 & IMF7 & IMF8 & IMF9 & IMF10 & IMF11 \\
\hline Filtred signal $y(t)$ & $\mathbf{0 . 8 6}$ & 0.53 & 0.16 & $\approx 0$ & $\approx 0$ & $\approx \mathbf{0}$ & $\approx 0$ & $\approx 0$ & $\approx 0$ & $\approx 0$ & $\approx 0$ \\
\hline
\end{tabular}

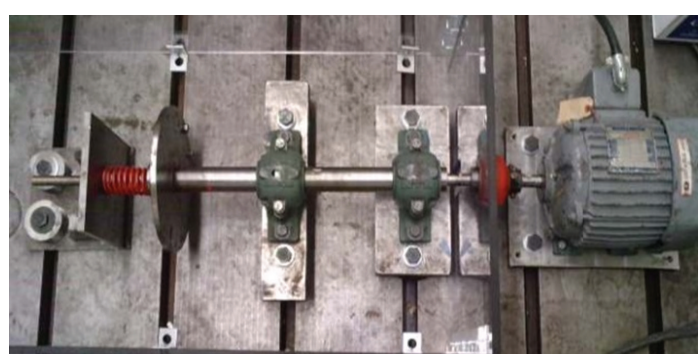

Figure 13: Test bench.

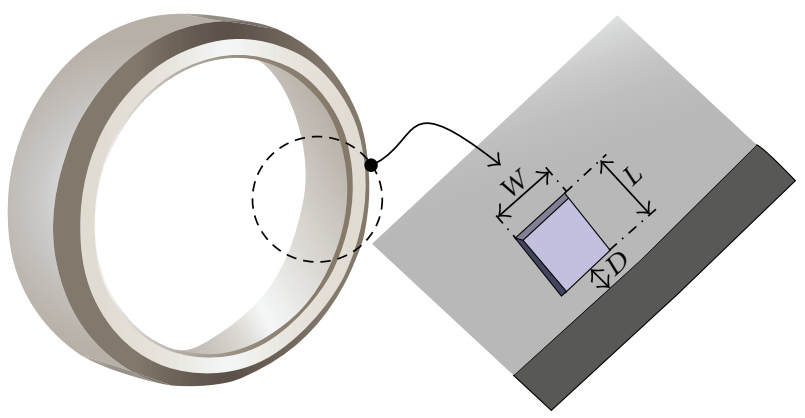

FIGURE 14: Defect in outer race.

signal. Hence, $\mathrm{IMF}_{1}$ was selected as the final resultant signal recovered from the raw signal.

The spectrum of TKEO for $\mathrm{IMF}_{1}$ (obtained by MEDEMD) is presented in Figure 12. We clearly detect the BPFO $(100 \mathrm{~Hz})$ due to the defect and its harmonics until $1200 \mathrm{~Hz}$. The hybrid method gives thus better results than the two others presented, previously.

5.3. Experimental Study. The test bench used in this study is shown in Figure 13. A shaft is supported by two bearings and connected to a motor with a flanged coupling bolted rubber. Two systems were investigated, one with a healthy bearing (D0) and the other with an artificially damaged bearing (on the outer race).

The defected bearing has a groove on the outer race as shown in Figure 14. The localized defects were created on outer race by using an electric discharge machine to keep their size and depth under control. The size of the artificial defect was about $200 \mu \mathrm{m}$ in depth and the width $(W)$ of the groove was $50 \mu \mathrm{m}$ for the first defect $(D 1)$ and $100 \mu \mathrm{m}$ for the second one $(D 2)$.

The measurements were conducted by using acoustic emission (AE) and vibration measurements [31]. The equipment for vibration data collection and acoustic emission is shown in Figure 15(a). They consist in an accelerometer with a sensitivity of $100 \mathrm{mV} / \mathrm{g}$ and an ultrasound detector (UE

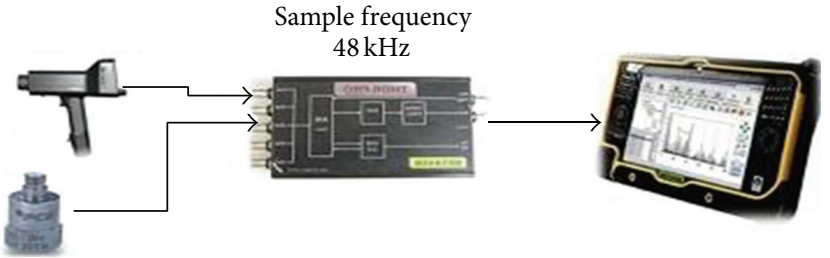

(a)

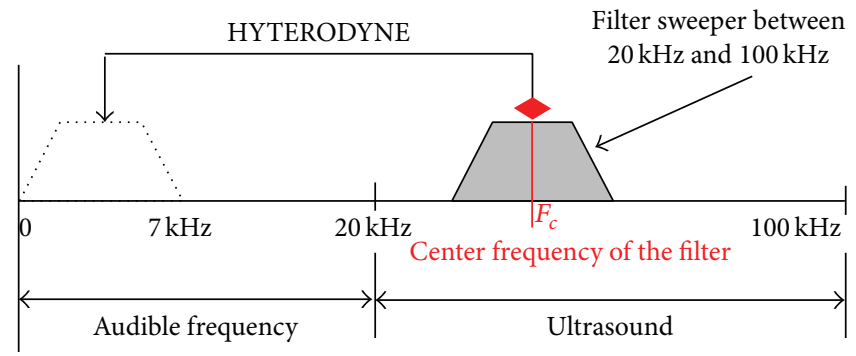

(b)

FIGURE 15: (a) Data acquisition system. (b) Heterodyne principle.

Systems UltraProb 10000). Both sensors are connected to an analogue digital converter (THOR Analyzer PRO) with a sampling frequency of $48 \mathrm{kHz}$. The latter is connected to a collector-analyzer BETAVIB. The ultrasonic sensor used in this study operates in the lower ultrasonic spectrum from $20 \mathrm{kHz}$ to $100 \mathrm{kHz}$. A heterodyne circuit converts the high frequency $\mathrm{AE}$ signal as detected by the transducer around a central frequency $F_{c}$ into an audible signal (0$7 \mathrm{kHz}$ ) (Figure 15(b)). The acoustic emission signals from the two bearings (healthy and faulty) were recorded at the central frequency of $F_{c}=30 \mathrm{kHz}$. The sensor filters the signal with a band $[27.5 \mathrm{kHz}-32.5 \mathrm{kHz}]$. After that, the heterodyne circuit converts the filtered signal into an audible signal. The heterodyned signal may then be recorded.

The acoustic emission and vibration signals from the healthy bearing $(D 0)$ and the two bearings ( $D 1$ and $D 2$ ) were recorded when rotating at two rotating speeds (300 and $600 \mathrm{rpm})$.

5.4. Experimental Application. The proposed method for signal processing was experimentally applied to the acoustic emission and vibration data.

5.4.1. Acoustic Emission. The EMD (method 2) and the MEDEMD (method 3) algorithms were applied and, to simplify the procedure, the IMF 1 was automatically selected. Tables 3 and 4 list the coefficient correlation between $\mathrm{IMF}_{1}$ obtained by applying EMD to the original signal and between the $\mathrm{IMF}_{1}$ after applying MED-EMD to the filtered signal with the three 
TABLE 3: Correlation value between $\mathrm{IMF}_{1}$ (obtained by EMD and MED-EMD) and the signal when rotating at $300 \mathrm{rpm}$.

\begin{tabular}{lccc}
\hline & & $300 \mathrm{rpm}$ & \\
& $0 \mu \mathrm{m}$ & $50 \mu \mathrm{m}$ & $100 \mu \mathrm{m}$ \\
\hline EMD & 0.53 & 0.40 & 0.42 \\
MED-EMD & 0.94 & 0.65 & 0.78 \\
\hline
\end{tabular}

TABLE 4: Correlation value between $\mathrm{IMF}_{1}$ (obtained by EMD and MED-EMD) and the signal when rotating at $600 \mathrm{rpm}$.

\begin{tabular}{lccc}
\hline & & $600 \mathrm{rpm}$ & \\
& $0 \mu \mathrm{m}$ & $50 \mu \mathrm{m}$ & $100 \mu \mathrm{m}$ \\
\hline EMD & 0.18 & 0.24 & 0.15 \\
MED-EMD & 0.38 & 0.39 & 0.72 \\
\hline
\end{tabular}

defect severity levels when rotating at $300 \mathrm{rpm}$ and $600 \mathrm{rpm}$, respectively. It may be noticed that the correlation value when applying MED-EMD increases whatever the defect size or rotational speed.

Figures 16, 17, and 18 display the spectrum of TKEO of $\mathrm{IMF}_{1}$ (obtained from MED-EMD: method 3) for the healthy bearing and for both defect sizes when rotating at $300 \mathrm{rpm}$.

The healthy bearing $(D 0)$ shows a flat spectrum as shown in Figure 16. When investigating a very small defect $(D 1)$, $\mathrm{BPFO}$ can be distinguished with its first harmonic (Figure 17). An increase of the peak amplitudes related to the BPFO frequency can be clearly noticed when the defect $D 2$ growths (Figure 18).

It can be noticed that the defect severity not only causes an increase in the amplitude, but also generates a lot of harmonics of the bearing frequency. Consequently, the key of diagnostic relies also on the number of harmonic of the bearing frequencies $[4,32]$.

When rotating at $600 \mathrm{rpm}$, the healthy bearing reveals a presence of the BPFO (Figure 19) witch small amplitudes, even if the bearing is assumed healthy. In fact, even a healthy bearing presents slight defects, such as surface roughness, which are excited when increasing the rotational speed.

For the defects $D 1$ as shown in Figure 20, the defect frequency BPFO can be clearly distinguished with its harmonics.

An increase of the peak amplitudes related to the BPFO frequency can be clearly noticed when the defect $D 2$ growths (Figure 21). It also can be noticed that the number of harmonics of the defect frequency increases when increasing the rotational speed.

5.4.2. Vibration Measurements. Tables 5 and 6 list coefficient correlation between $\mathrm{IMF}_{1}$ obtained by applying EMD to the original signal and between the $\mathrm{IMF}_{1}$ after applying MEDEMD to the filtered signal when rotating at $300 \mathrm{rpm}$ and $600 \mathrm{rpm}$, respectively. We have the same conclusion than for acoustic emission. The correlation value when applying MED-EMD increases whatever the defect size or rotational speed.

Figures 22 and 23 display the vibration spectrum of TKEO of $\mathrm{IMF}_{1}$ (obtained from MED-EMD: method 3) for both

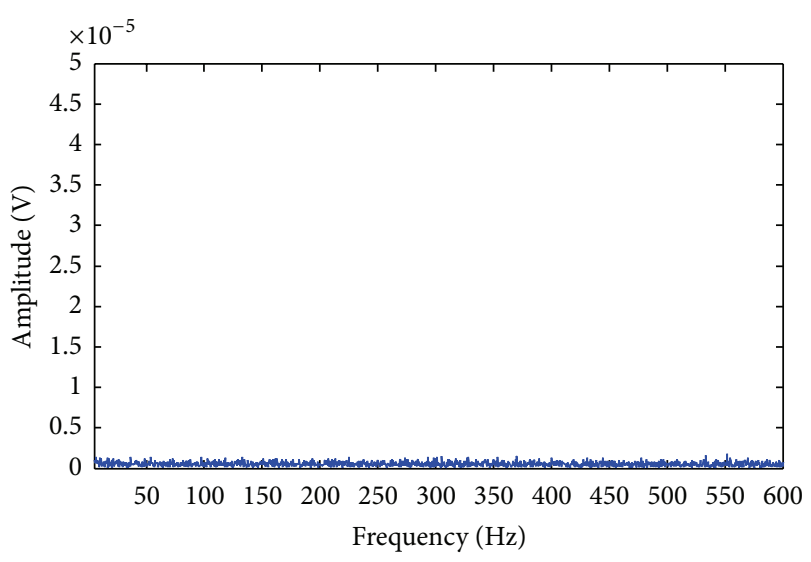

FIGURE 16: Acoustic emission spectrum of TKEO of IMF $_{1}$ obtained by applying MED-EMD to D0 at speed $300 \mathrm{rpm}$.

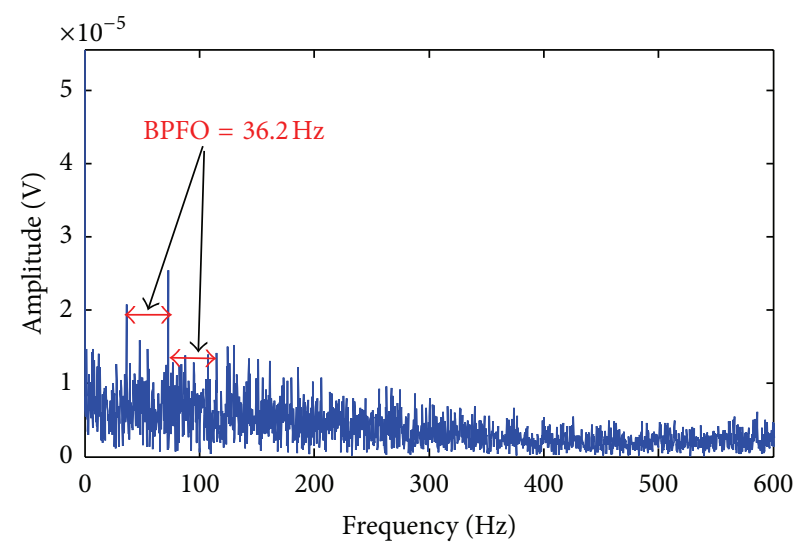

FIgURE 17: Acoustic emission spectrum of TKEO of $\mathrm{IMF}_{1}$ obtained by applying MED-EMD to $D 1$ at speed $300 \mathrm{rpm}$.

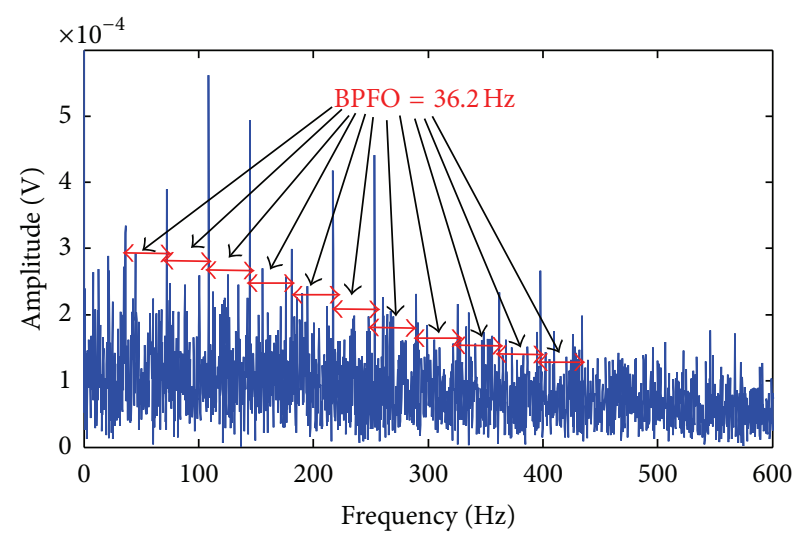

FIGURE 18: Acoustic emission spectrum of TKEO of IMF $_{1}$ obtained by applying MED-EMD to D2 at speed $300 \mathrm{rpm}$.

defect sizes when rotating at $300 \mathrm{rpm}$. When investigating a very small defect $(D 1)$, the fundamental of BPFO cannot be distinguished so clearly (Figure 22) than with acoustic emission (Figure 17). 


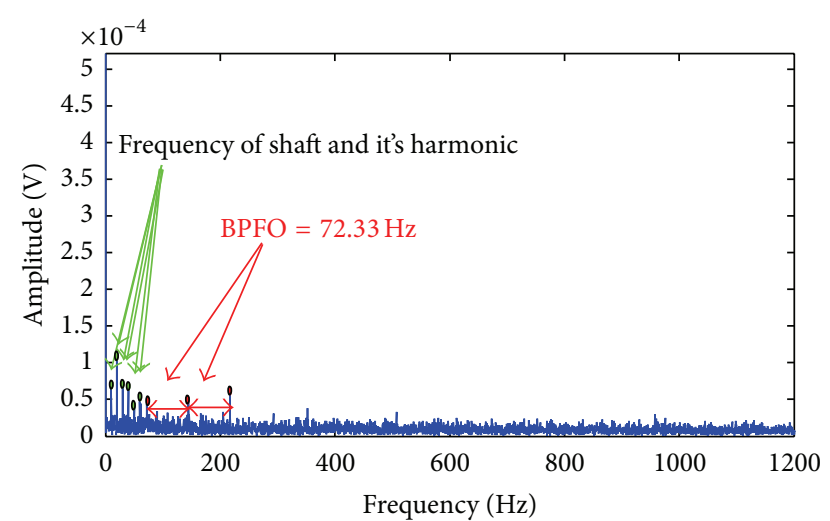

FIGURE 19: Acoustic emission spectrum of TKEO of IMF $_{1}$ obtained by applying MED-EMD to $D 0$ case at speed $600 \mathrm{rpm}$.

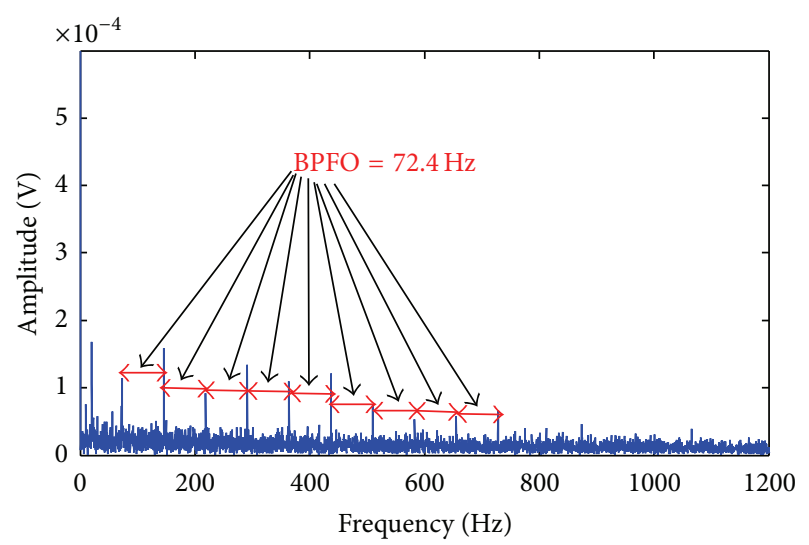

Figure 20: Acoustic emission spectrum of TKEO of IMF $_{1}$ obtained by applying MED-EMD to $D 1$ case at speed $600 \mathrm{rpm}$.

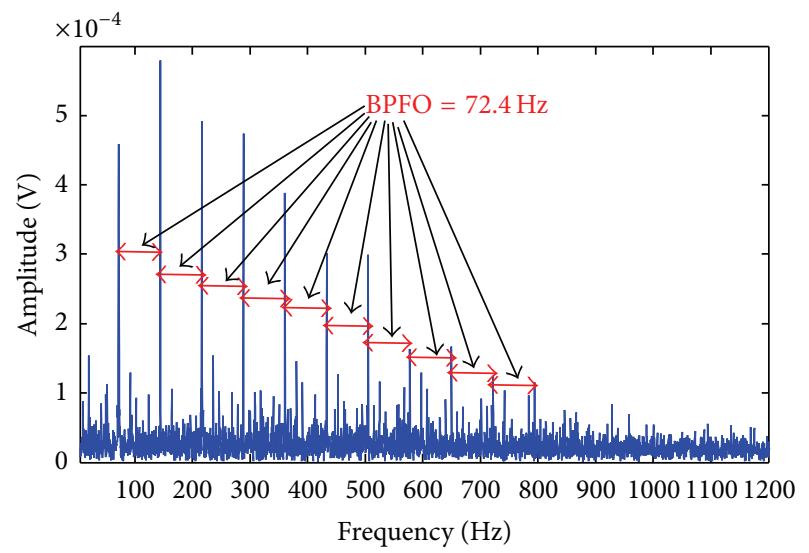

FIGURE 21: Acoustic emission spectrum of TKEO of IMF $_{1}$ obtained by applying MED-EMD to $D 2$ case at speed $600 \mathrm{rpm}$.

An increase of the peak amplitudes related to the BPFO frequency can be clearly noticed when the defect $D 2$ growths (Figure 23).

For the defects $D 1$ as shown in Figure 24, the frequency $\mathrm{BPFO}$ can be distinguished with its harmonics when rotating at $600 \mathrm{rpm}$. An increase of the peak amplitudes related to the

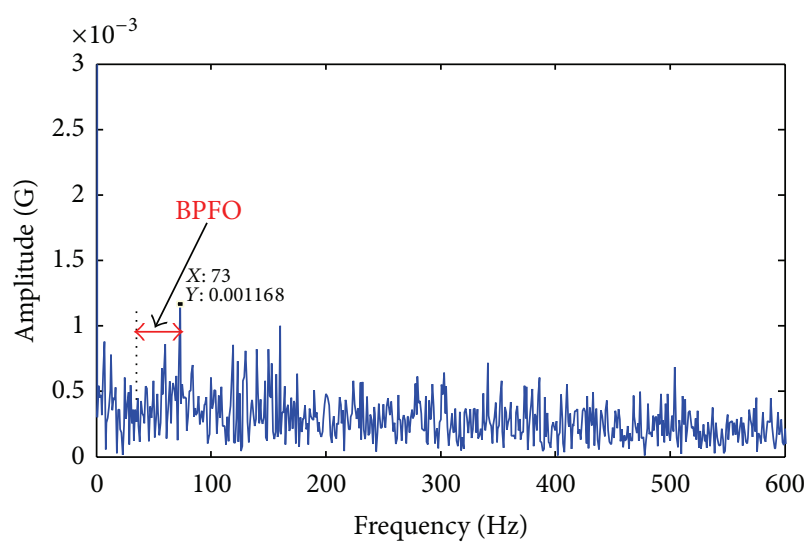

FIGURE 22: Vibration spectrum of TKEO of $\mathrm{IMF}_{1}$ obtained by applying MED-EMD to $D 1$ at speed $300 \mathrm{rpm}$.

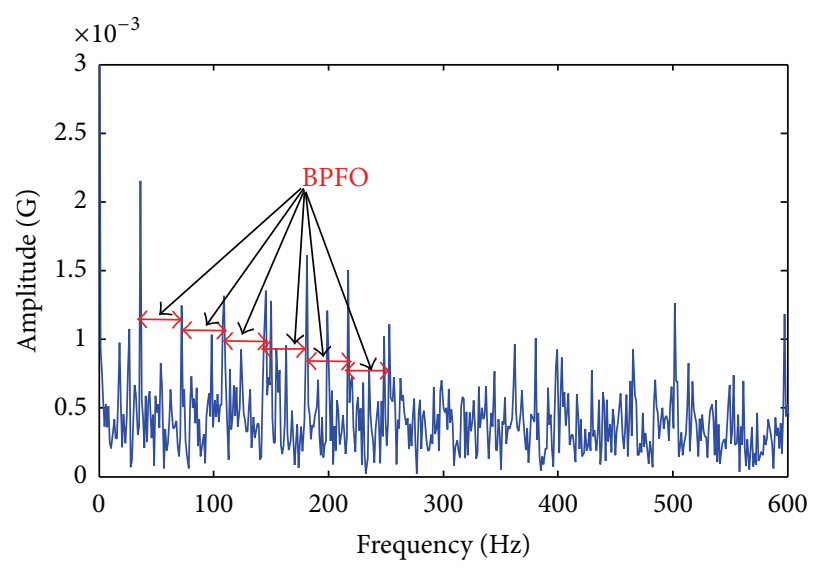

FIGURE 23: Vibration spectrum of TKEO of $\mathrm{IMF}_{1}$ obtained by applying MED-EMD to D2 at speed $300 \mathrm{rpm}$.

TABLE 5: Correlation value between $\mathrm{IMF}_{1}$ (obtained by EMD and MED-EMD) and the signal when rotating at $300 \mathrm{rpm}$.

\begin{tabular}{lcc}
\hline & \multicolumn{3}{c}{$300 \mathrm{rpm}$} \\
& $50 \mu \mathrm{m}$ & $100 \mu \mathrm{m}$ \\
\hline EMD & 0.45 & 0.25 \\
MED-EMD & 0.65 & 0.27 \\
\hline
\end{tabular}

TABLE 6: Correlation value between $\mathrm{IMF}_{1}$ (obtained by EMD and MED-EMD) and the signal when rotating at $600 \mathrm{rpm}$.

\begin{tabular}{lcc}
\hline & \multicolumn{2}{c}{$600 \mathrm{rpm}$} \\
& $50 \mu \mathrm{m}$ & $100 \mu \mathrm{m}$ \\
\hline EMD & 0.31 & 0.26 \\
MED-EMD & 0.75 & 0.41 \\
\hline
\end{tabular}

BPFO frequency and to its harmonics can be clearly noticed when the defect $D 2$ growths (Figure 25).

Furthermore, comparing acoustic emission and vibration measurement reveals that the identification of defects is clearer and better well defined by acoustic emission than from vibration measurements. 


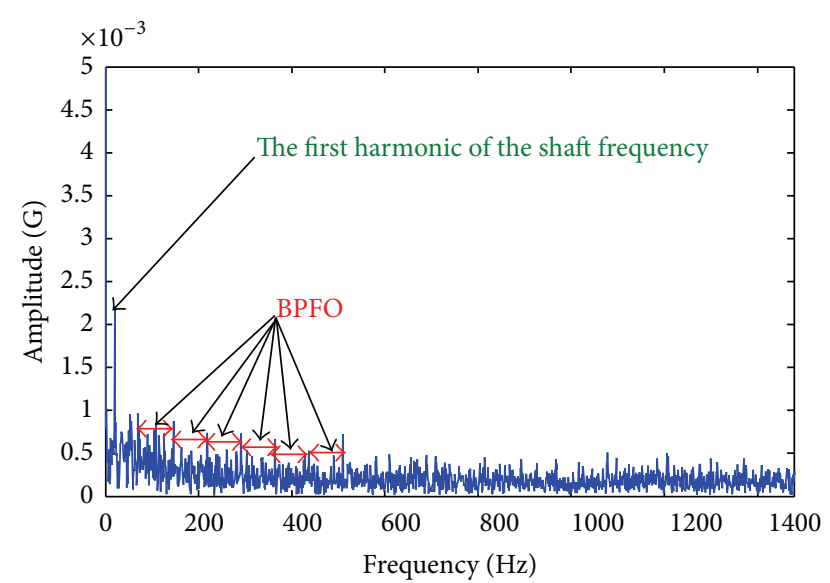

FIGURE 24: Vibration spectrum of TKEO of $\mathrm{IMF}_{1}$ obtained by applying MED-EMD to $D 1$ at speed $600 \mathrm{rpm}$.

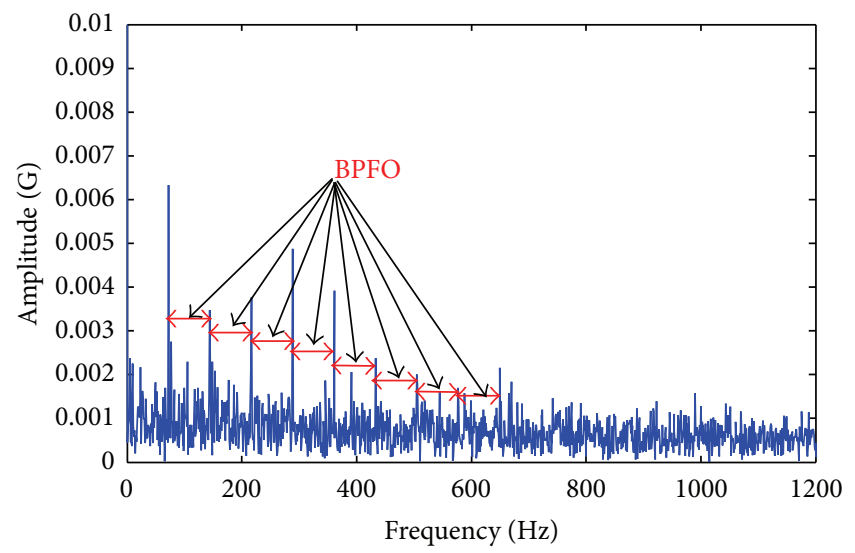

FIGURE 25: Vibration spectrum of TKEO of $\mathrm{IMF}_{1}$ obtained by applying MED-EMD to D2 at speed $600 \mathrm{rpm}$.

\section{Conclusions}

In this paper, a new approach for fault diagnosis of bearings is presented. The algorithm involves a simple transformation and a spectral analysis step. Three methods are compared. The selected method is hybrid. First, the signal is filtered by using EMD. After that, the obtained signal is decomposed into several IMFs. The study of the correlation coefficient between each IMF and the original signal allows for automatically selecting the better IMF. In our study, the first component $\mathrm{IMF}_{1}$, which represents the highest frequency components, was considered to accomplish the diagnosis by using the TKEO metric in order to compute the instantaneous amplitude. The method has been both applied to simulated signals and experimental signals obtained by acoustic emission and vibration measurement to validate the approach. The results have shown the effectiveness of the method. The fundamental advantage of the proposed method relies on the fact that no prior knowledge about the structural dynamics is required. The results have shown that the proposed method can effectively reveal the frequency and its associated harmonics of the faulty bearing.

\section{Conflict of Interests}

The authors declare that there is no conflict of interests regarding the publication of this paper.

\section{Acknowledgments}

The financial support of NSERC (Natural Sciences and Engineering Research Council of Canada), FQRNT (Fonds Québecois de la Recherche sur la Nature et les Technologies), MITACS Canada, and Pratt \& Whitney Canada is gratefully acknowledged.

\section{References}

[1] P. D. McFadden and J. D. Smith, "Vibration monitoring of rolling element bearings by the high-frequency resonance technique-a review," Tribology International, vol. 17, no. 1, pp. 3-10, 1984.

[2] P. W. Tse, Y. H. Peng, and R. Yam, "Wavelet analysis and envelope detection for rolling element bearing fault diagnosistheir effectiveness and flexibilities," Journal of Vibration and Acoustics, Transactions of the ASME, vol. 123, no. 3, pp. 303-310, 2001.

[3] J. Altmann and J. Mathew, "Multiple band-pass autoregressive demodulation for rolling-element bearing fault diagnosis," Mechanical Systems and Signal Processing, vol. 15, no. 5, pp. 963977, 2001.

[4] M. Thomas, Reliability, Predictive Maintenance and Machinery Vibration, Presses de l'Université du Québec, 2011, (French).

[5] J. Antoni and R. B. Randall, "The spectral kurtosis: application to the vibratory surveillance and diagnostics of rotating machines," Mechanical Systems and Signal Processing, vol. 20, no. 2, pp. 308-331, 2006.

[6] Y. Imaouchen, M. Thomas, and R. Alkama, "Detection of bearing defects by combining Hilbert Transform to Kurtogram," in Proceedings of the 2nd International Conference on Maintenance, Gestion, Logistic and Electrotechnic (CIMGLE'12), pp. 1-6, Oran, Algeria, November 2012.

[7] T. Barszcz and A. Jabłoński, "A novel method for the optimal band selection for vibration signal demodulation and comparison with the Kurtogram," Mechanical Systems and Signal Processing, vol. 25, no. 1, pp. 431-451, 2011.

[8] H. Qiu, J. Lee, J. Lin, and G. Yu, "Wavelet filter-based weak signature detection method and its application on rolling element bearing prognostics," Journal of Sound and Vibration, vol. 289, no. 4-5, pp. 1066-1090, 2006.

[9] H. Qiu, J. Lee, J. Lin, and G. Yu, "Robust performance degradation assessment methods for enhanced rolling element bearing prognostics," Advanced Engineering Informatics, vol. 17, no. 3-4, pp. 127-140, 2003.

[10] N. G. Nikolaou and I. A. Antoniadis, "Demodulation of vibration signals generated by defects in rolling element bearings using complex shifted Morlet wavelets," Mechanical Systems and Signal Processing, vol. 16, no. 4, pp. 677-694, 2002.

[11] J. Lin and M. J. Zuo, "Gearbox fault diagnosis using adaptive wavelet filter," Mechanical Systems and Signal Processing, vol. 17, no. 6, pp. 1259-1269, 2003.

[12] J. F. Kaiser, "On a simple algorithm to calculate the 'energy' of a signal," in Proceedings of the International Conference on Acoustics, Speech, and Signal Processing, pp. 381-384, April 1990. 
[13] A. Potamianos and P. Maragos, "A comparison of the energy operator and the Hilbert transform approach to signal and speech demodulation," Signal Processing, vol. 37, no. 1, pp. 95120, 1994.

[14] P. Maragos, J. F. Kaiser, and T. F. Quatieri, "On amplitude and frequency demodulation using energy operators," IEEE Transactions on Signal Processing, vol. 41, no. 4, pp. 1532-1550, 1993.

[15] P. Maragos, J. F. Kaiser, and T. F. Quatieri, "Energy separation in signal modulations with application to speech analysis," IEEE Transactions on Signal Processing, vol. 41, no. 10, pp. 3024-3051, 1993.

[16] C. Junsheng, Y. Dejie, and Y. Yu, "The application of energy operator demodulation approach based on EMD in machinery fault diagnosis," Mechanical Systems and Signal Processing, vol. 21, no. 2, pp. 668-677, 2007.

[17] Z. Yuping, L. Hui, and B. Lihong, "Adaptive instantaneous frequency estimation based on EMD and TKEO," in Proceedings of the 1st International Congress on Image and Signal Processing (CISP '08), pp. 60-64, May 2008.

[18] M. Liang and I. S. Bozchalooi, "An energy operator approach to joint application of amplitude and frequency-demodulations for bearing fault detection," Mechanical Systems and Signal Processing, vol. 24, no. 5, pp. 1473-1494, 2010.

[19] G. F. Bin, J. J. Gao, X. J. Li, and B. S. Dhillon, "Early fault diagnosis of rotating machinery based on wavelet packetsempirical mode decomposition feature extraction and neural network," Mechanical Systems and Signal Processing, vol. 27, no. 1, pp. 696-711, 2012.

[20] T. Kidar, M. Thomas, R. Guilbault, and M. El Badaoui, "Comparison between the sensitivity of LMD and EMD algorithms for early detection of gear defects," Mechanics \& Industry, Cambridge University Press, vol. 14, no. 2, pp. 121-127, 2013.

[21] T. Kidar, M. Thomas, M. El Badaoui, and R. Guilbault, "Application of time descriptors to the modified Hilbert transform of empirical mode decomposition for early detection of gear defects," in Proceedings of the 2nd International Conference on Condition Monitoring of Machinery in Non-Stationary Operations (CMMNO '12), pp. 471-480, Hammamet, Tunisia, March 2012.

[22] M. Kedadouche, M. Thomas, and A. Tahan, "Empirical mode decomposition of acoustic emission for early detection of bearing defects," in Proceedings of the 3rd International Conference on Condition Monitoring of Machinery in Non-Stationary Operations (CMMNO '13), pp. 1-11, Ferrara, Italy, 2013.

[23] Z. Feng, M. Liang, Y. Zhang, and S. Hou, "Fault diagnosis for wind turbine planetary gearboxes via demodulation analysis based on ensemble empirical mode decomposition and energy separation," Renewable Energy, vol. 47, pp. 112-126, 2012.

[24] H. Li, L. Fu, and Z. Li, "Fault detection and diagnosis of gear wear based on Teager-Huang transform," in Proceedings of the 1st IITA International Joint Conference on Artificial Intelligence (JCAI '09), pp. 663-666, April 2009.

[25] H. Li, Y. Zhang, and H. Zheng, "Bearing fault detection and diagnosis based on order tracking and Teager-Huang transform," Journal of Mechanical Science and Technology, vol. 24, no. 3, pp. 811-822, 2010.

[26] N. Sawalhi, R. B. Randall, and H. Endo, "The enhancement of fault detection and diagnosis in rolling element bearings using minimum entropy deconvolution combined with spectral kurtosis," Mechanical Systems and Signal Processing, vol. 21, no. 6, pp. 2616-2633, 2007.
[27] H. Endo and R. B. Randall, "Enhancement of autoregressive model based gear tooth fault detection technique by the use of minimum entropy deconvolution filter," Mechanical Systems and Signal Processing, vol. 21, no. 2, pp. 906-919, 2007.

[28] R. A. Wiggins, "Minimum entropy deconvolution," Geoexploration, vol. 16, no. 1-2, pp. 21-35, 1978.

[29] G. L. McDonald, Q. Zhao, and M. J. Zuo, "Maximum correlated Kurtosis deconvolution and application on gear tooth chip fault detection," Mechanical Systems and Signal Processing, vol. 21, pp. 2616-2633, 2007.

[30] Y.-T. Sheen, "A complex filter for vibration signal demodulation in bearing defect diagnosis," Journal of Sound and Vibration, vol. 276, no. 1-2, pp. 105-119, 2004.

[31] M. Kedadouche, M. Thomas, and A. Tahan, "Monitoring bearings by acoustic emission: a comparative study with vibration techniques for early detection," in Proceedings of the 30th Seminar on Machinery Vibration (CMVA '12), pp. 1-17, Niagara Falls, Canada, October 2012.

[32] M. Thomas, J. Masounave, T. M. Dao, C. T. Le Dinh, and F. Lafleur, "Rolling element bearing degradation and vibration signature relationship," in Proceedings of the 2nd International Conference on Surveillance Methods and Acoustical and Vibratory Diagnosis, vol. 1, pp. 267-277, SFM, 1995. 

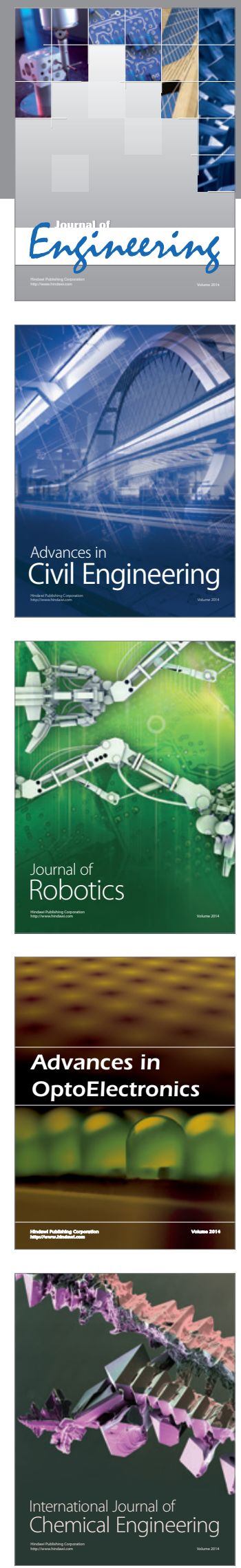

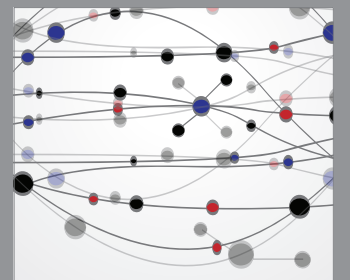

The Scientific World Journal
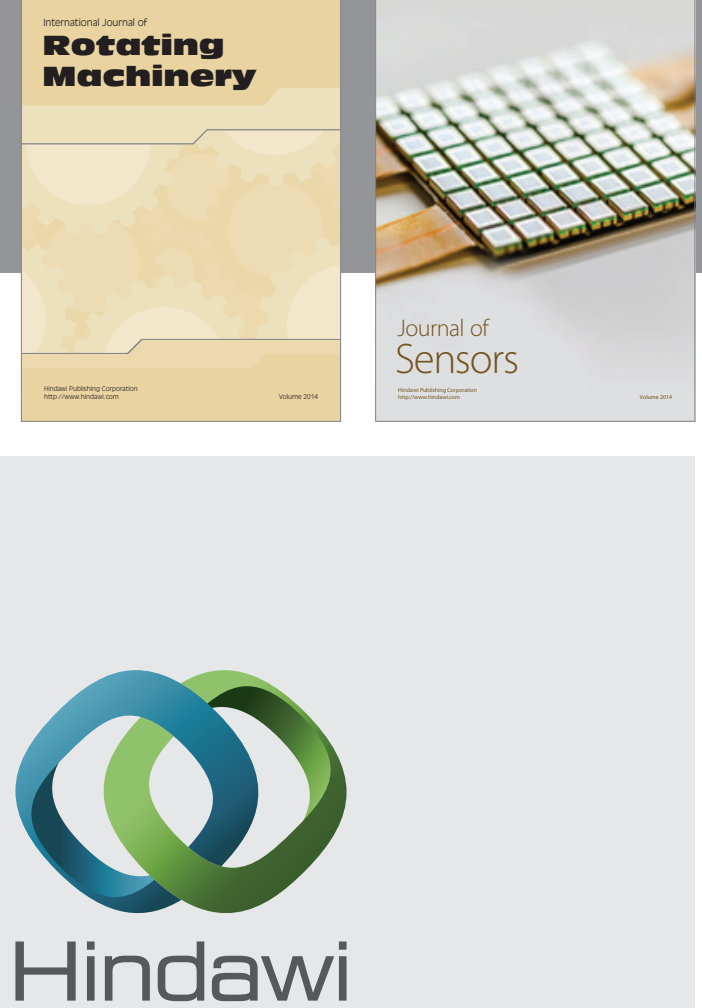

Submit your manuscripts at http://www.hindawi.com
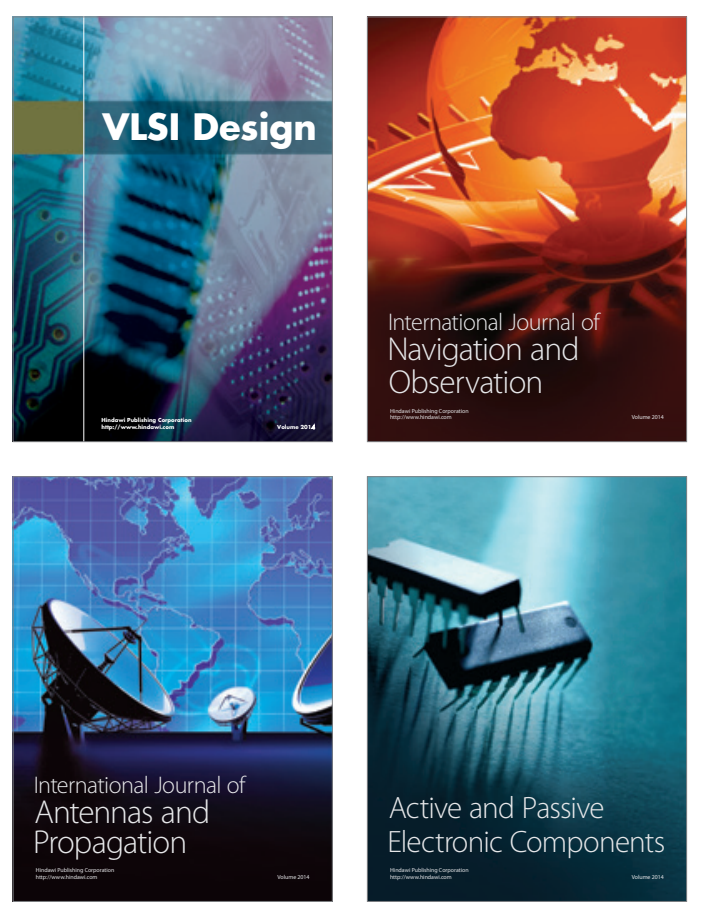
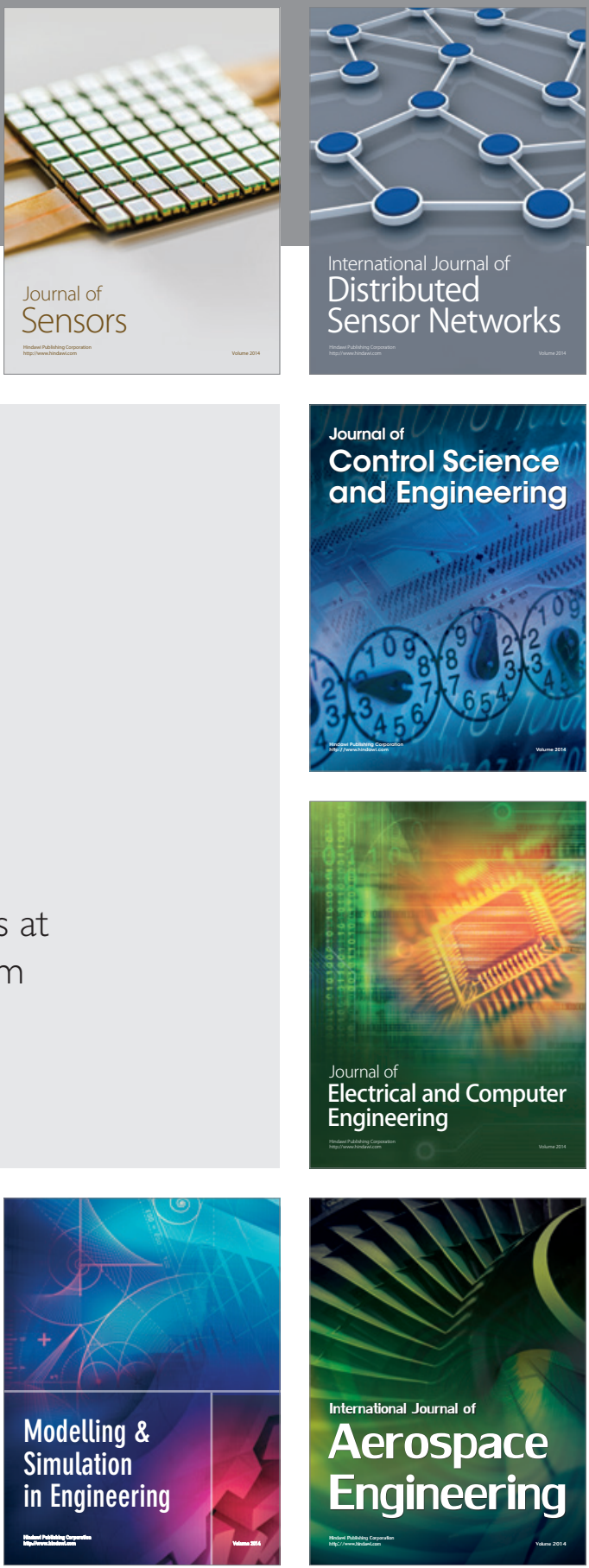

Journal of

Control Science

and Engineering
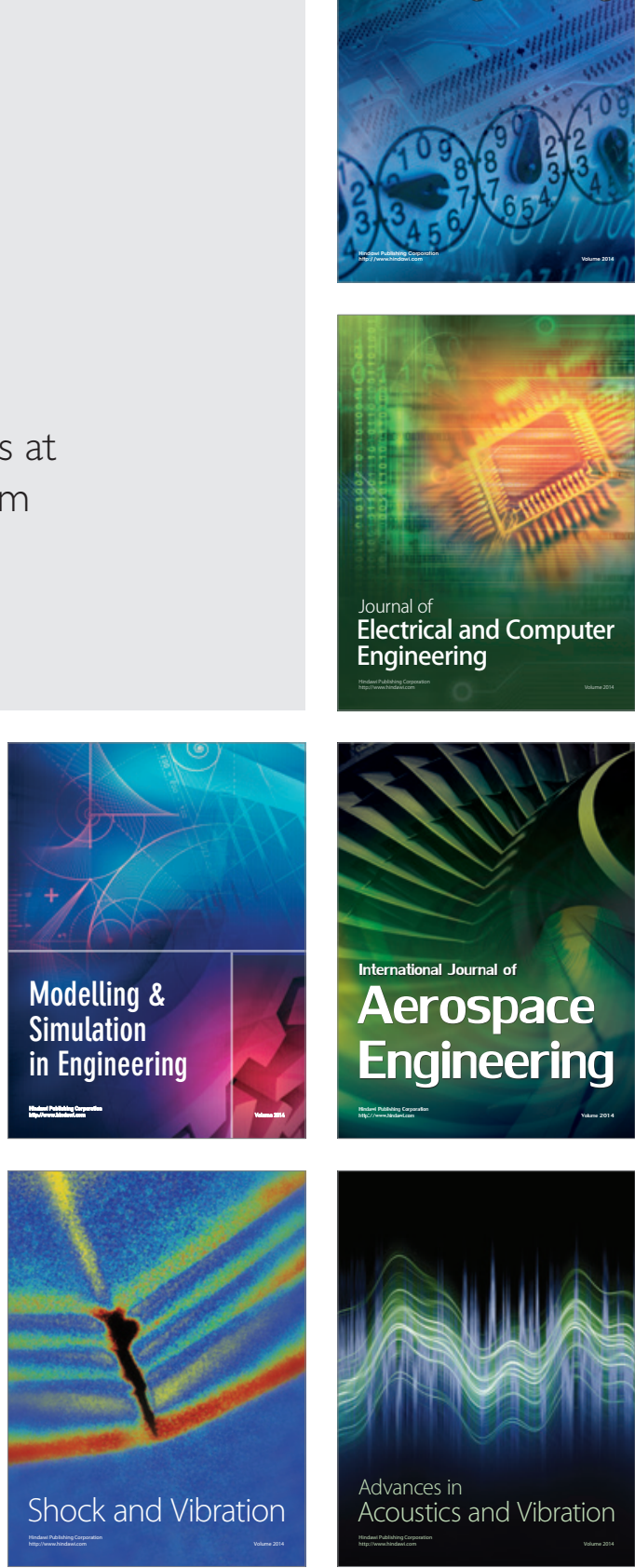